\title{
Improving left ventricular segmentation in four- dimensional flow MRI using intramodality image registration for cardiac blood flow analysis
}

Vikas Gupta, Mariana Bustamante, Alexandru Fredriksson, Carljohan Carlhäll and Tino Ebbers

The self-archived postprint version of this journal article is available at Linköping University Institutional Repository (DiVA):

http:// urn.kb.se/ resolve?urn=urn:nbn:se:liu:diva-143887

N.B.: When citing this work, cite the original publication.

Gupta, V., Bustamante, M., Fredriksson, A., Carlhäll, C., Ebbers, T., (2018), Improving left ventricular segmentation in four-dimensional flow MRI using intramodality image registration for cardiac blood flow analysis, Magnetic Resonance in Medicine, 79(1), 554-560. https:// doi.org/ 10.1002/ mrm.26674

Original publication available at:

https:// doi.org/ 10.1002/ mrm.26674

Copyright: Wiley

http:/ / eu.wiley.com/WileyCDA/ 


\title{
Improving left ventricular segmentation in 4D Flow MRI using intra-modality image registration for cardiac blood flow analysis
}

\author{
Vikas Gupta*†a,b $^{*}$, Mariana Bustamante ${ }^{\dagger a, b}$, Alexandru Fredriksson ${ }^{\mathrm{a}}$, Carl-Johan Carlhäll $^{\mathrm{a}, \mathrm{b}, \mathrm{c}}$, and Tino Ebbers ${ }^{\mathrm{a}, \mathrm{b}}$ \\ ${ }^{a}$ Division of Cardiovascular Medicine, Department of Medical and Health Sciences, Linköping University, Linköping, 58185, Sweden \\ ${ }^{b}$ Center for Medical Image Science and Visualization (CMIV), Linköping University, Linköping, 58185, Sweden \\ ${ }^{c}$ Department of Physiology and Department of Medical and Health Sciences, Linköping University, Linköping, 58185, Sweden
}

Purpose: Assessment of blood flow in the left ventricle (LV) using four-dimensional (4D) Flow MRI requires accurate LV segmentation that is often hampered by the low contrast between blood and the myocardium. The purpose of this work is to improve left-ventricular segmentation in 4D Flow MRI for reliable blood flow analysis.

Method: The LV segmentations are first obtained using morphological cine-MRI with better in-plane resolution and contrast, and then aligned to 4D Flow MRI data. This alignment is, however, not trivial due to inter-slice misalignment errors caused by patient motion and respiratory drift during breath-hold based cine-MRI acquisition. A robust image registration based framework is proposed to mitigate such errors automatically. Data from 20 subjects, including healthy volunteers and patients, was used to evaluate its geometric accuracy and impact on blood flow analysis.

Results: High spatial correspondence was observed between manually and automatically aligned segmentations, and the improvements in alignment compared to uncorrected segmentations were significant $(P<0.01)$. Blood flow analysis from manual and automatically corrected segmentations did not differ significantly $(P>0.05)$.

Conclusion: Our results demonstrate the efficacy of the proposed approach in improving left-ventricular segmentation in 4D Flow MRI, and its potential for reliable blood flow analysis.

Key words: 4D Flow MRI, image registration, blood flow analysis, cardiology, MRI

\section{INTRODUCTION}

Assessing pulsatile blood flow through the left ventricle (LV) of the heart is vital for understanding the cardiovascular function in health and disease. Four-dimensional (4D) Flow MRI has been developed and successfully used for such an assessment [1. However, it requires accurate geometric identification of underlying cardiac or vascular anatomy that is often poorly visible even to an experienced clinician, mainly due to low resolution and poor contrast between blood and the myocardium. One of the commonly used solutions to obtain reliable segmentations is the utilization of higher in-plane resolution and better contrast in routinely acquired multi-slice cine balanced steady-state free precession (cine-bSSFP) MR images 2]. The desired segmentation is first delineated on the cine-bSSFP images and then manually aligned to the corresponding 4D Flow MRI data. Although such a procedure provides a close estimate of the required anatomical geometry, it is quite tedious, slow, and prone to inter- and intra-observer bias. Moreover, alignment errors may also be introduced due to sub-optimal breath-holding [3, 4] leading to respiratory drift during the acquisition of multiple slices of the cinebSSFP image 4, 5]. The respiratory-induced motion of the heart was studied in detail by McLeish et al. 3] and the application of image registration was suggested to mitigate such misalignment errors. Inaccuracies in the segmentation used for the analysis of blood flow can affect the results significantly, and could be ground for the exclusion of data [6. 7].

Image registration methods have been successfully used to solve motion correction problems in a multitude of applications 8 . Typically, these methods receive a pair of images as input, usually referred to as "reference" and "target", and generate as output a transformation that brings them into a common spatial alignment [8]. Long-axis (LAX) cine-bSSFP images have often been used as reference for this purpose 9 11, but with the assumption that they are free of motion artifacts. Several other methods exist for motion correction in cine-bSSFP images [12, but lack a suitable reference image for reliable alignment. In contrast to cine-bSSFP images, 4D Flow MR images are acquired with navigator gating, hence, no breath-holds are required during their acquisition. They are, consequently, less prone to respiration induced motion, and can be an effective reference for image registration.

The purpose of this study is to investigate the feasibility of robust and automatic image registration between breath-hold acquired morphological cine-bSSFP and respiratory-gated 4D Flow MRI to improve the accuracy of left-ventricular segmentation, and hence, the subsequent blood flow analysis. 


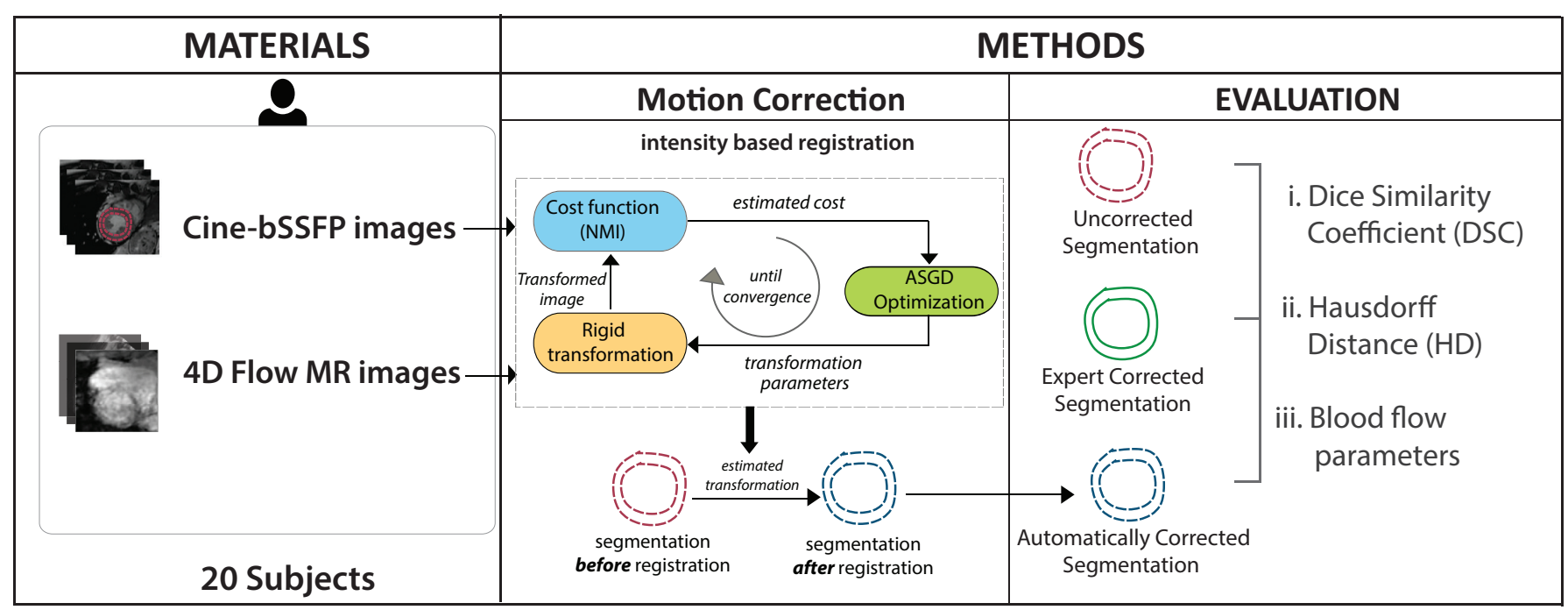

Figure 1: An overview of the materials and methods

\section{METHODS}

\section{Study population}

The proposed method was evaluated in 20 subjects, including 10 healthy volunteers and 10 patients with ischemic cardiomyopathy (CMP) or idiopathic dilated CMP. The healthy volunteers had no history of prior or current cardiovascular disease or cardiac medication. Exclusion criteria for the patients were: significant ventricular arrhythmia, heart rate $<40 \mathrm{bpm}$ or $>100 \mathrm{bpm}$, and more than mild to moderate left-sided valvular disorder. The research was performed in line with the Helsinki declaration, and was approved by the regional ethics board. All subjects gave written informed consent.

\section{Image acquisition and pre-processing}

The MRI examinations were performed on a clinical 3T Philips Ingenia scanner (Philips Healthcare, Best, the Netherlands). All subjects were injected with a Gadolinium contrast agent (Magnevist, Bayer Schering Pharma AG) prior to the acquisition for a late-enhancement study.

Cine-bSSFP images were acquired at end-expiratory breath-holds, resulting in 30 time-frames over the cardiac cycle. A short-axis stack with resolution of $1 \times 1 \mathrm{~mm}^{2}$, and slice thickness of $8 \mathrm{~mm}$ was acquired. Two-, three-, and four-chamber long-axis views were also obtained with the same in-plane resolution.

4D Flow MRI examinations were performed during freebreathing, using a navigator gated gradient-echo pulse sequence with interleaved three-directional flow-encoding and retrospective vector cardiogram controlled cardiac gating. Scan parameters included: Candy cane view adjusted to cover both ventricles, velocity encoding $120 \mathrm{~cm} / \mathrm{s}, 40$ timeframes, spatial resolution $2.7 \times 2.7 \times 2.8 \mathrm{~mm}^{3}$, and elliptical k-space acquisition. Typical scan time was 10-15 min. The 4D Flow MRI data were corrected for concomitant gradient fields on the MRI scanner. Phase wraps were corrected offline using temporal phase unwrapping method [13, and background phase errors were corrected using a weighted $2^{\text {nd }}$ order polynomial fit to the static tissue [14.

\section{Region of interest (ROI)}

As the organs surrounding the heart (e.g., lungs, liver) in both 4D Flow MR and cine-bSSFP images might interfere with the registration of the heart, a region of interest encompassing only the left ventricle (LV) was calculated before the registration. The following steps describe the automatic selection of a square ROI with a fixed edge length, $L$ (80 mm, determined empirically), for each image slice:

1. Obtain a binary mask using thresholding based on Otsu's method [15].

2. Find the largest region thresholded, due to a high probability of it containing the heart.

3. Obtain the centroid, $C$, of this region.

4. Define a square mask with $C$ at its center, and $L$ as the length of its edges.

\section{Image registration}

The target (cine-bSSFP) images of the heart were spatially aligned to the reference (corresponding ROI of the 4D Flow MR magnitude component) using a rigid transformation, $T$. Calculation of the three-dimensional area of intersection between the cine-bSSFP and 4D Flow MR magnitude image was implemented as an interpolation of the $4 \mathrm{D}$ Flow MR image at each location of the cine-bSSFP image's voxels. The estimated optimal transformation $\hat{T}$ was obtained by minimizing a cost function (or maximizing a similarity measure), $\phi$, as described in the following equation [16]:

$$
\hat{T}=\arg \min _{T} \phi\left(T\left(I_{t}\right), I_{r}\right),
$$


where, $I_{r}$ and $I_{t}$ represent the reference and target images, respectively.

An intensity based registration approach was adopted as it does not require a prior feature extraction (landmark positions, surfaces, etc.), and is less susceptible to noise and outliers [17. Since the underlying signal in both cine-bSSFP and 4D Flow MR comes from MRI and both types of images represent the same anatomical regions, normalized cross-correlation (NCC) and normalized mutual information (NMI) [18] were tested as the cost function. For two supposedly similar signals, NCC measures their correlation while NMI measures the similarity between their distributions. These were computed using equations 2 and 5 , respectively.

$$
\operatorname{NCC}\left(I_{r}, I_{\hat{t}}\right)=\frac{\sum_{x_{i} \in \Omega_{r}}\left(I_{r}\left(x_{i}\right)-\bar{I}_{r}\right)\left(I_{\hat{t}}\left(x_{i}\right)-\bar{I}_{\hat{t}}\right)}{\sqrt{\sum_{x_{i} \in \Omega_{r}}\left(I_{r}\left(x_{i}\right)-\bar{I}_{r}\right)^{2} \sum_{x_{i} \in \Omega_{r}}\left(I_{\hat{t}}\left(x_{i}\right)-\bar{I}_{\hat{t}}\right)^{2}}},
$$

where,

$$
\begin{aligned}
\bar{I}_{r} & =\frac{1}{\left|\Omega_{r}\right|} \sum_{x_{i} \in \Omega_{r}} I_{r}\left(x_{i}\right), \text { and } \\
\bar{I}_{\hat{t}} & =\frac{1}{\left|\Omega_{r}\right|} \sum_{x_{i} \in \Omega_{r}} I_{t}\left(T\left(x_{i}\right)\right) \\
\operatorname{NMI}\left(I_{r}, I_{\hat{t}}\right) & =\frac{\sum_{t \in H_{t}} \sum_{r \in H_{r}} p(r, \hat{t}) \log _{2}\left(p_{r}(r) p_{t}(\hat{t})\right)}{\sum_{t \in H_{t}} \sum_{r \in H_{r}} p(r, \hat{t}) \log _{2} p(r, \hat{t})}
\end{aligned}
$$

where, $H_{r}$ and $H_{t}$ are sets of regularly spaced intensity bin centers; $p$ is the discrete joint probability; and $p_{r}$ and $p_{t}$ are the marginal discrete probabilities of the reference and target images.

In order to make the registration both unsupervised and computationally efficient, an optimization method with preferably no free parameters is required. To this end, we used an adaptive stochastic gradient descent (ASGD) strategy [19] that performs computationally efficient random data sampling for gradient calculation at each iteration. Moreover, unlike standard gradient descent strategies, it obviates the need to select a step size a priori by using an adaptive prediction. It was shown to be very fast and robust in finding the optimal step sizes using a stochastic process [19].

Further gains in computational and convergence efficiency were obtained by implementing a coarse-to-fine multi-resolution strategy that helped in avoiding local minima and achieving fast convergence. An overview of the method and its validation is provided in Figure 1

\section{Evaluation}

The registration method was evaluated by measuring geometric accuracy using organ segmentation, which has shown to be more reliable than an intensity based comparison 20, combined with an assessment of the impact on blood flow analysis on 4D Flow MRI.

Three variants of manual LV segmentations were compared for the evaluation: uncorrected $\left(S_{\text {uncorr }}\right)$, automatically corrected $\left(S_{\text {auto }}\right)$, and expert corrected $\left(S_{\text {expert }}\right)$. $S_{\text {uncorr }}$ refers to the original LV segmentations, created de novo on cine-bSSFP images in the entire cohort by an expert (AGF) with five years of experience. To account for misalignments caused by respiratory motion between the slices of the cine-bSSFP images, these segmentations $\left(S_{\text {uncorr }}\right)$ were rigidly transformed to match the LV anatomy in the 4D Flow images by: (a) the same expert (AGF) and (b) using the transformation, $\hat{T}$ (equation 1), from the proposed registration. Thus obtaining expert and automatically corrected segmentations, $S_{\text {expert }}$ and $S_{\text {auto }}$ respectively.

Geometric accuracy of the proposed method was assessed by measuring the spatial correspondence using Dice Similarity Coefficient (DSC) 21] and Hausdorff distance (HD) 22 between the corrected $\left(S_{\text {auto }}\right)$ and expert $\left(S_{\text {expert }}\right)$ segmentations. DSC is defined as the ratio of the intersection between two segmentations to their average area (equation 6) and its values range between 0 (no overlap) and 1 (complete overlap).

$$
\operatorname{DSC}\left(S_{\text {expert }}, S_{\text {auto }}\right)=\frac{2\left|S_{\text {expert }} \cap S_{\text {auto }}\right|}{\left|S_{\text {expert }}\right|+\left|S_{\text {auto }}\right|}
$$

HD between the two segmentations were computed by measuring the maximum distance between their corresponding points:

$$
\operatorname{HD}\left(S_{\text {expert }}, S_{\text {auto }}\right)=\max _{i, j}\left(d\left(e_{i}, S_{\text {auto }}\right), d\left(c_{j}, S_{\text {expert }}\right)\right)
$$

where $\left[e_{1}, e_{2}, \ldots, e_{n}\right]$ and $\left[c_{1}, c_{2}, \ldots, c_{n}\right]$ refer to the points in $S_{\text {expert }}$ and $S_{\text {auto }}$, respectively, and $d$ is the Euclidean distance between the points. DSC and HD were similarly computed between $S_{\text {uncorr }}$ and $S_{\text {expert }}$.

In addition, pathline analysis of the $4 \mathrm{D}$ flow MRI data was performed using the uncorrected, expert, and corrected segmentations on the 4D Flow MR images to study the impact of the correction on LV blood flow analysis. The evaluation was performed using an established method to separate the LV end-diastolic volume into functional flow components 23]. Following this method, the LV was segmented at end-diastolic and end-systolic time-points. Pathlines were computed forwards and backwards in time from the end-diastolic volume. Thereafter, the end-diastolic LV blood volume is separated into four flow components, based on the blood's transit through the LV chamber. The evaluation was performed on inflow, outflow, end-diastolic and end-systolic flow volumes in the LV, which were computed by combining the appropriate flow components.

\section{Statistical analyses}

To determine whether the improvements observed with the proposed method were significant, the results from the 


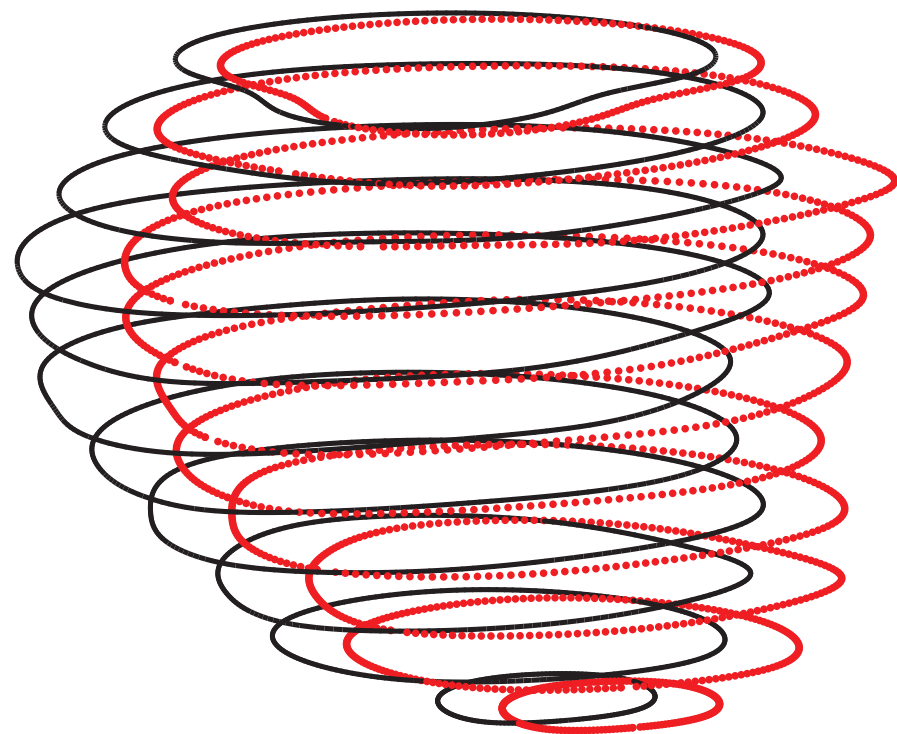

(a) Uncorrected vs Expert corrected

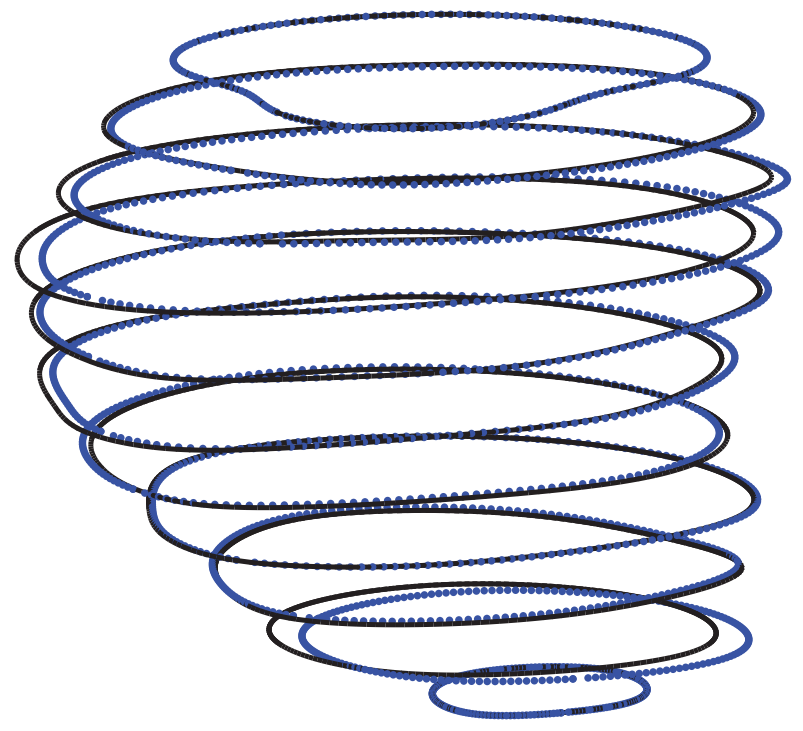

(b) Automatically vs Expert corrected

Figure 2: An example of the differences between the uncorrected (dotted red) and the automatically corrected (dotted blue) segmentations when compared with the expert corrected segmentations (solid black). Only LV endocardial segmentations are shown for clarity. The order of the slices is from base (top) to apex (bottom). Significantly high distances $(\mathrm{p}<0.01)$ were observed between the expert and the uncorrected segmentations.

uncorrected and corrected segmentations were compared with the expert's results. A 2-sided Wilcoxon signed rank test was used to test the significance $(P<0.01)$.

\section{Implementation}

An open-source software, Elastix [16], was used for image registration, while the evaluation was performed using custom tools built with MATLAB, Release 2016a (The MathWorks, Inc., Natick, Massachusetts, United States).

\section{RESULTS}

\section{Geometric accuracy}

Figure 2 shows an example of the distances between LV segmentations before and after correction with the proposed method. The baseline or reference for this comparison is the set of corresponding expert corrected segmentations. While the distance between the uncorrected and expert corrected segmentations is large for various slices, it is greatly decreased after the correction as shown in Supporting Table S1. As different regions of the LV may experience variable motion during breath-holding, we analyzed the segmentations before and after correction in the basal, mid-ventricular, and apical regions of the LV. The results of this analysis is presented in Figure 3. It can be seen that correction can be necessary in all slices, but that in general the largest corrections are necessary in the apical slices. The $S_{\text {auto }}(N M I)$ resulted in improved DSC and HD measures in all sections, while the $S_{\text {auto }}(N C C)$ generated low DSC values and high HD values in the apical slices.

\section{Impact on blood flow analysis}

The evaluation of results originating from the flow pathline analysis can be seen in Figures 4 and 5 . Mean and variation of differences increased significantly $(P<0.01)$ with higher displacements for uncorrected segmentations, but remained consistently low for corrected segmentations. When compared to the blood flow using the expert corrected segmentation, significant deviations were observed with the uncorrected segmentation, while little to no differences were observed with the automatically corrected segmentation (figure 5).

\section{Discussion}

This study presents an automatic method for the correction of misalignment errors in LV segmentations to enable accurate flow analysis from 4D Flow MR images. Although the proposed method is evaluated only on LV, it can potentially be used also for the other cardiac chambers. To the best of our knowledge, this is the first method for improving LV segmentation in this type of MR images. The results demonstrate the accuracy and robustness of the proposed registration approach in providing an accurate estimate of left ventricular anatomy, which is vital for reliable blood flow analysis.

\section{Geometric accuracy}

The geometric accuracy of the proposed method was assessed with two measures: DSC and HD. While DSC is an intuitive measure, it is often insensitive to fine details. $\mathrm{HD}$, on the other hand, is highly sensitive to small regions of poor segmentation. When used together, they offered 


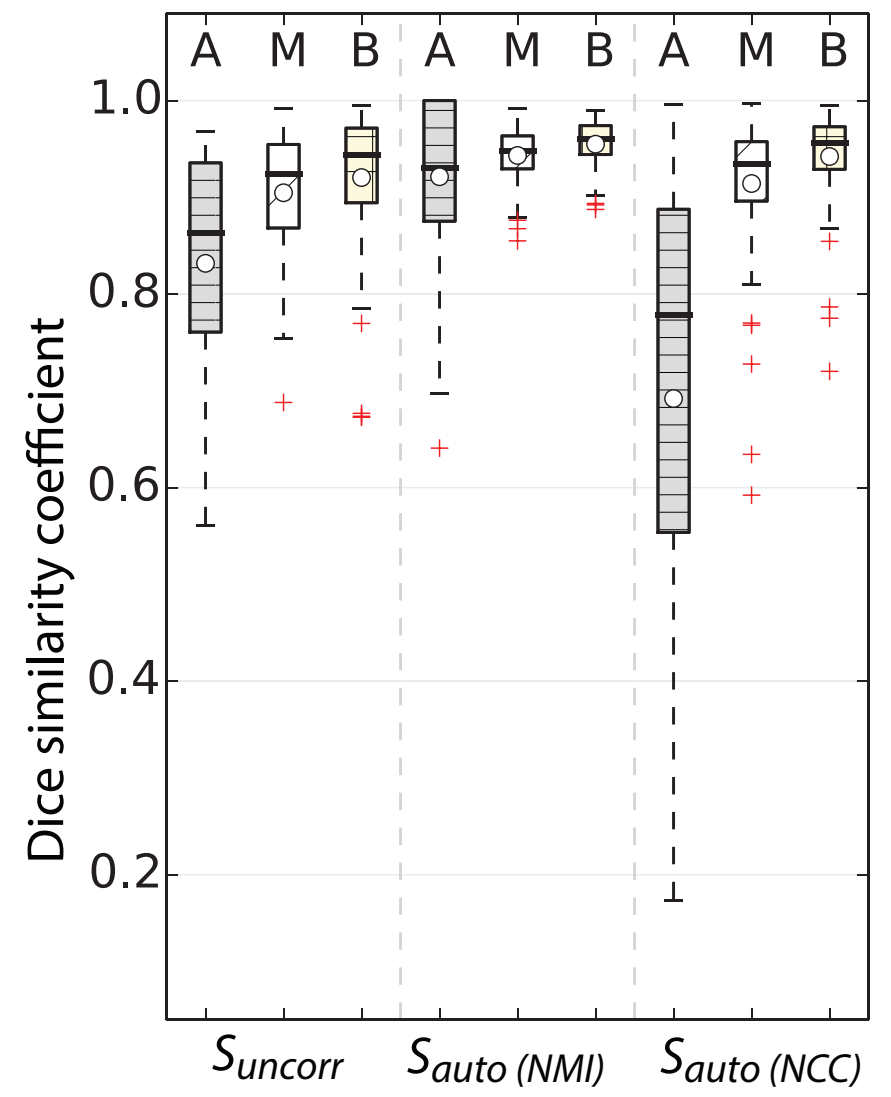

(a) Dice similarity coefficient (DSC)

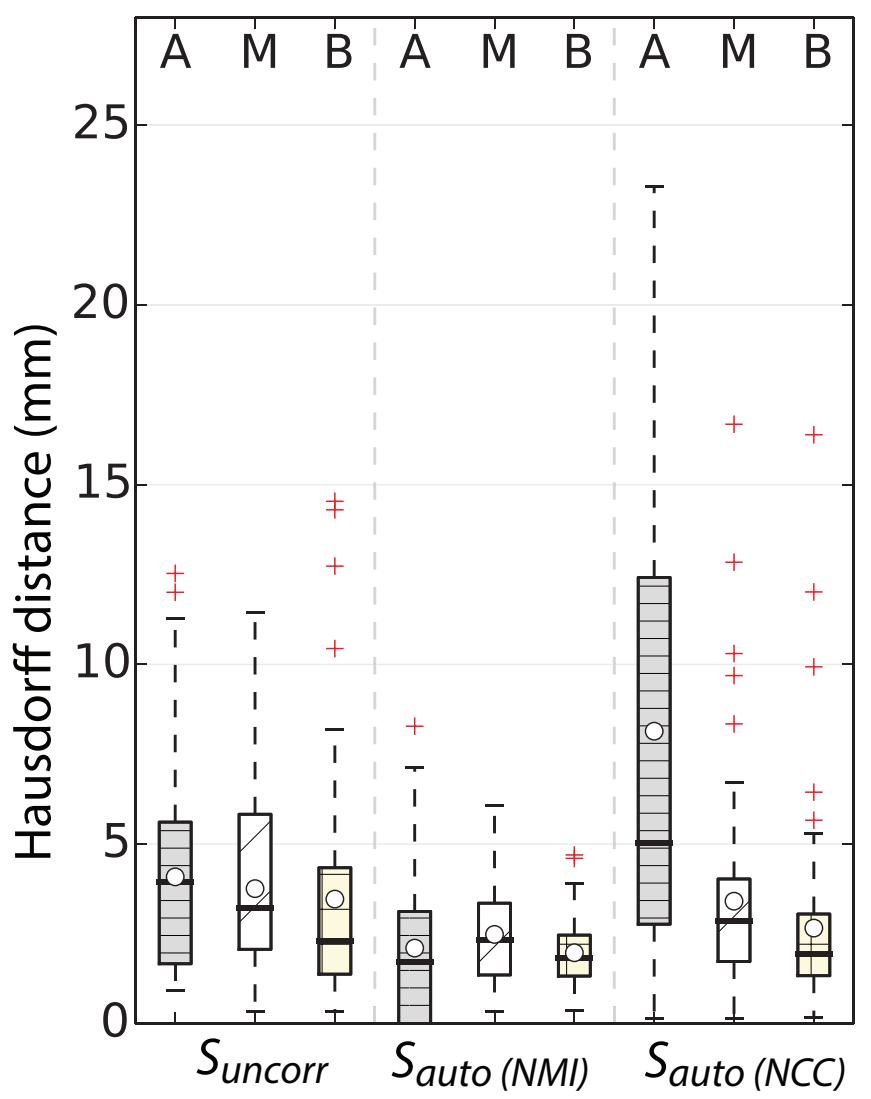

(b) Hausdorff distance (HD)

Figure 3: Dice similarity coefficient (DSC) and Hausdorff distance (HD) for different regions (A: Apical, M: Mid-ventricular, B:Basal) of the left ventricle. These measures show the accuracy of uncorrected $\left(S_{\text {uncorr }}\right)$ and automatically corrected segmentations, $S_{\text {auto }}$ with respect to the expert corrected segmentations $\left(S_{\text {expert }}\right)$. $S_{a u t o}(N M I)$ and $S_{a u t o}(N C C)$ were obtained using NCC and NMI as the cost functions, respectively. The value of DSC varies between 0 and 1 , where 1 refers to a complete overlap. The horizontal line inside the boxes indicate the median and the circles refer to the means, while their lower and upper edges report first and third quartiles, respectively. The whiskers reflect confidence intervals and are defined as the most extreme data points (1.5 times the interquartile range) not considered outliers ('+').

a robust means for assessing the quality of registration taking into account both the global shape and finer details of the expected segmentation. Both measures showed that NMI performed better than NCC. This points to a low correlation between absolute intensities in cine-bSSFP and 4D Flow MR images, but a high statistical dependence that is measured by NMI. Of particular importance were the low variances in DSC and HD (Figure 3 across different regions and in the entire cohort, thus demonstrating the robustness with respect to a heterogeneous set of data. In general, the performance was slightly better in basal and mid-ventricular regions compared to the apical regions. This was expected, since determining the exact location of the left ventricle near the apex is difficult due to thinner wall and more trabeculation, even for a clinical expert. Furthermore, DSC and HD values do not worsen after motion correction (Figure 3), which indicates that the overlap and distances between the expert and automatically corrected segmentations either remain the same or become better, but do not become worse.

\section{Impact on blood flow analysis}

Blood flow parameters estimated using expert corrected and uncorrected segmentations showed significant $(P<0.01)$ differences whereas a similar comparison with automatically corrected segmentations resulted in insignificant $(P>0.05)$ differences. A further breakdown of differences based on multiple levels of misalignments/displacements (Figure 4 showed considerably high discrepancies with uncorrected segmentations and consistently low differences with corrected segmentations. The lower differences at displacements less than $2 \mathrm{~mm}$ could be attributed to the relatively higher velocities of blood flow 4, when compared to the motion artifacts. The impact of high displacements on blood flow analysis using pathlines is shown in figure 5. High discrepancies in the different blood flow components were observed, especially near the septum, when the segmentations are uncorrected. Automatically corrected segmentations, in contrast, provided the same components on par with expert corrected segmentations. These results support our hypothesis that accurate LV segmentations are necessary for a reliable blood flow analysis. 


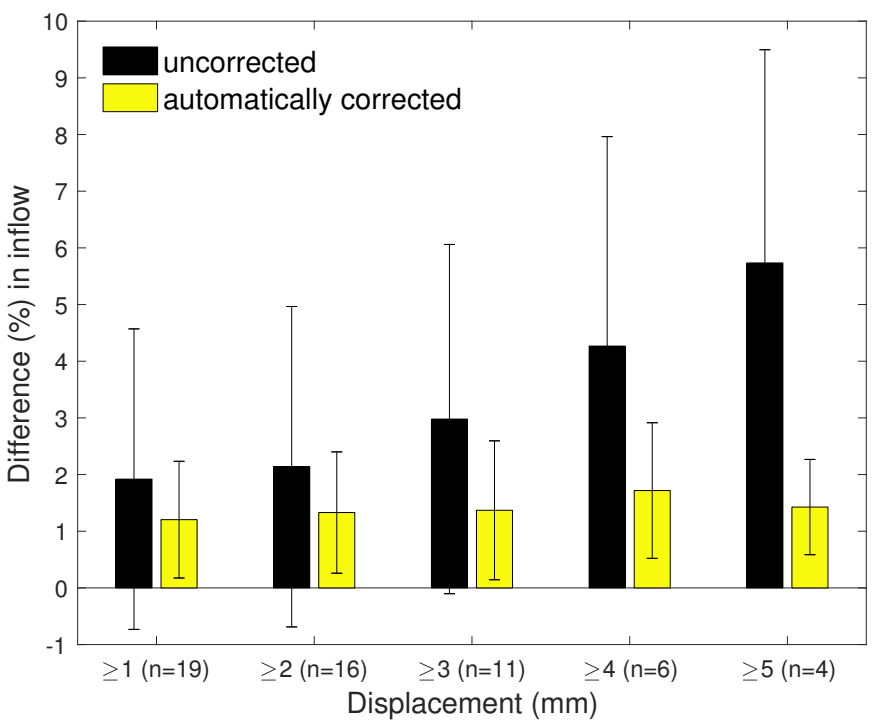

(a) Inflow

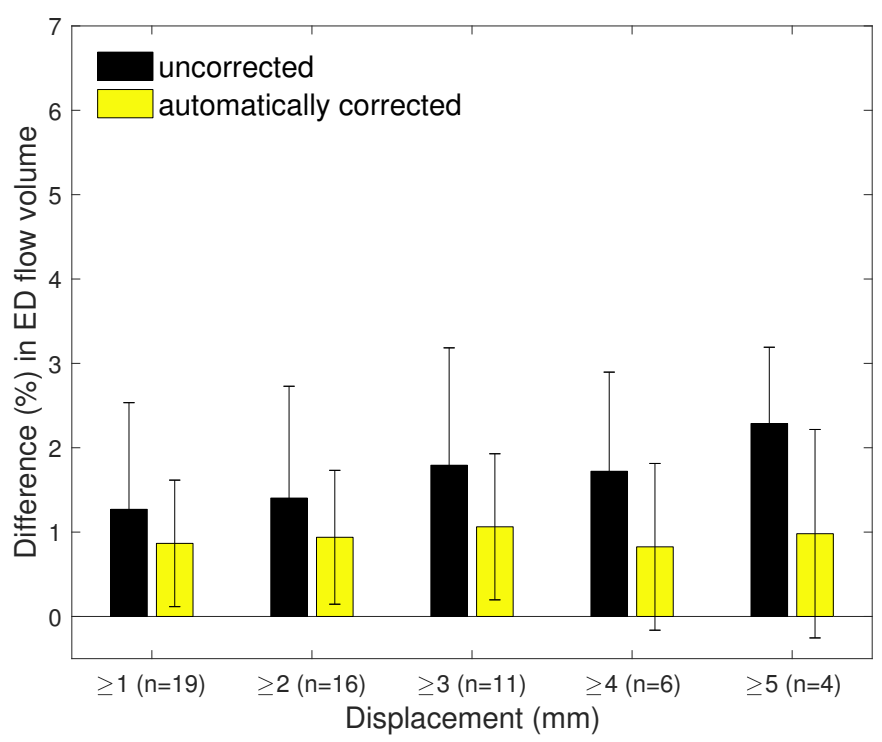

(c) End-diastolic blood flow

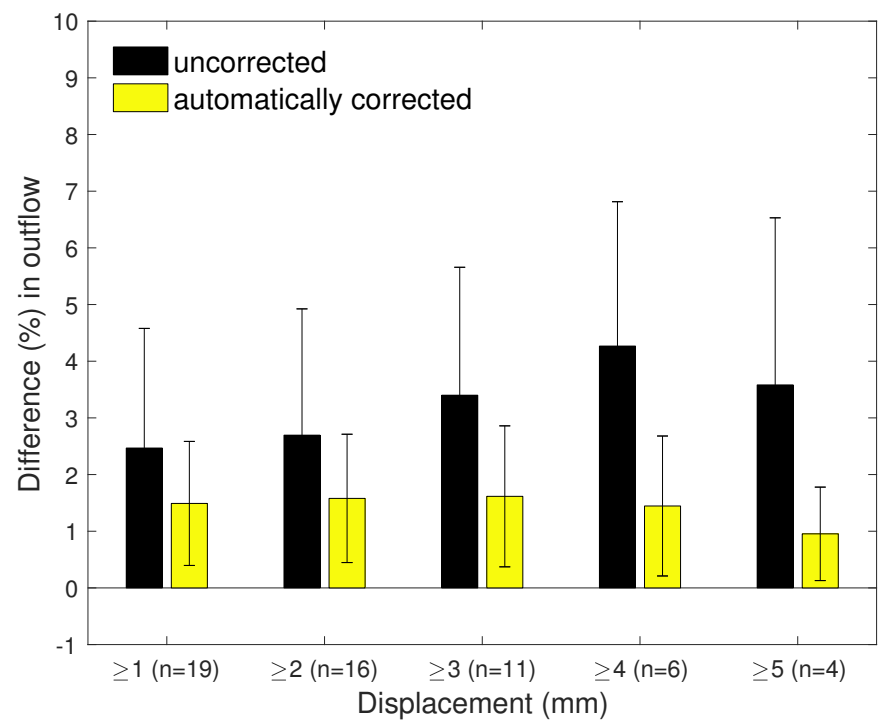

(b) Outflow

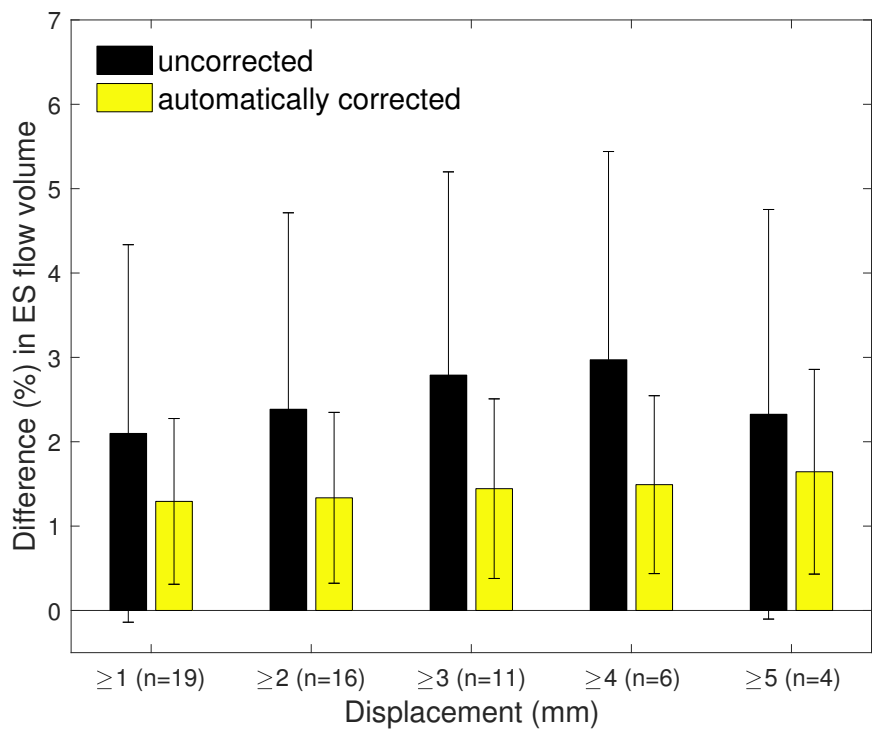

(d) End-systolic blood flow

Figure 4: The differences (\%) in inflow, outflow, end-diastolic flow volume, and end-systolic flow volume estimated for uncorrected and automatically corrected segmentations with respect to the expert corrected segmentations. The height of the bar shows mean and the error bar shows standard deviation. These differences are shown for subjects with breath-hold motion (displacements) greater than 1, 2, 3, 4 and 5 mm. The number (n) in the parenthesis shows the number of subjects for the corresponding group.

\section{Advantages}

Previous registration-based methods for segmentation correction in cine-bSSFP images have often used LAX views as the reference 9 10]11. Using this approach in our application would have improved the cine-bSSFP images, but would not have solved misalignment between the cine-bSSFP and 4D Flow MRI data. Furthermore, by using the LAX views as reference, the useful data for the registration is limited to the intersection of these images, which typically consists of only one line per LAX image. In the proposed method, the inclusion of a full three- dimensional image as the reference significantly increases the amount of data that can be used by the registration method to calculate a suitable transformation. We use a rigid in-plane registration, which is fast, robust, and avoids deformation of important clinical information in the images. Adding more degrees of freedom to the rigid transformation, as proposed in [24, could potentially improve the results at the cost of an increased processing time and potentially decreased robustness. However, our results on blood flow analysis show that our simpler transformation is sufficient and we do not expect any major improvements by adding 

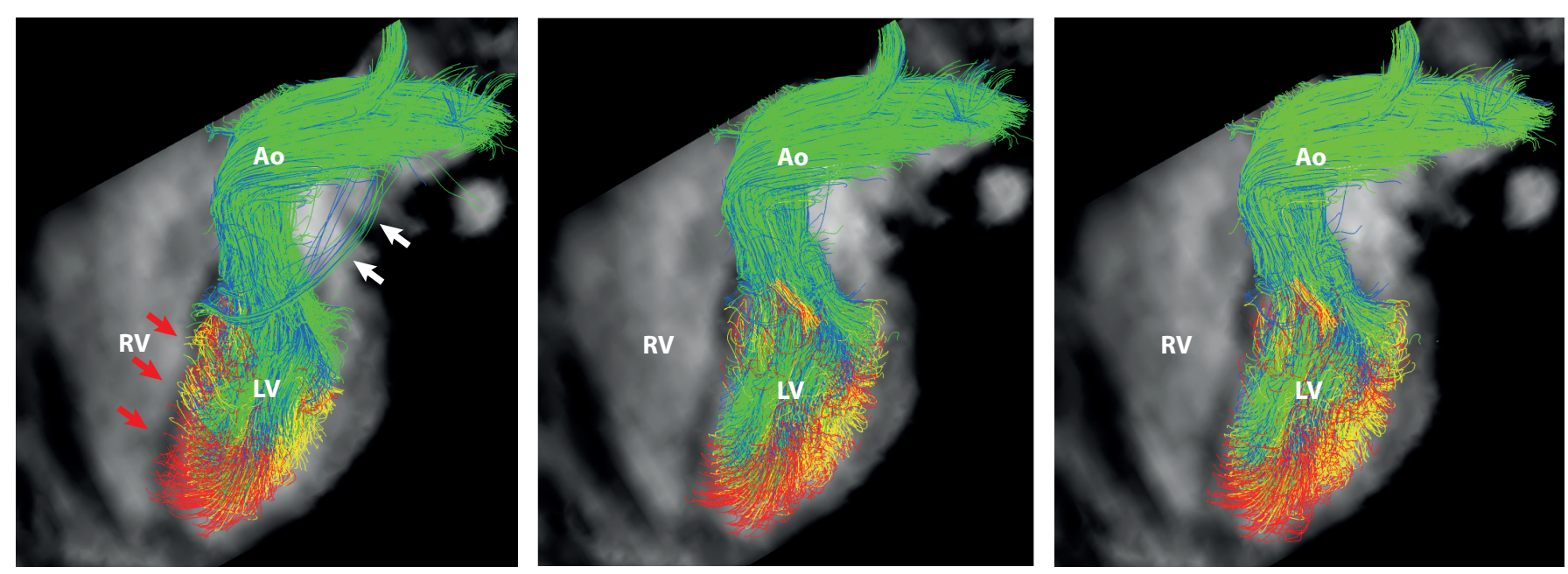

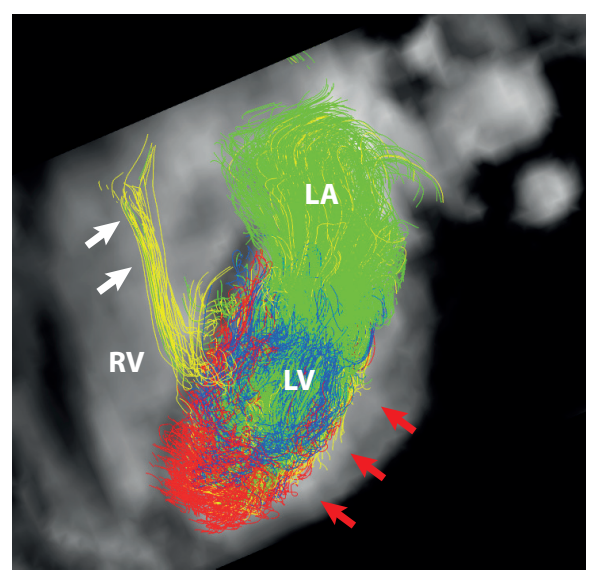

(a) Uncorrected

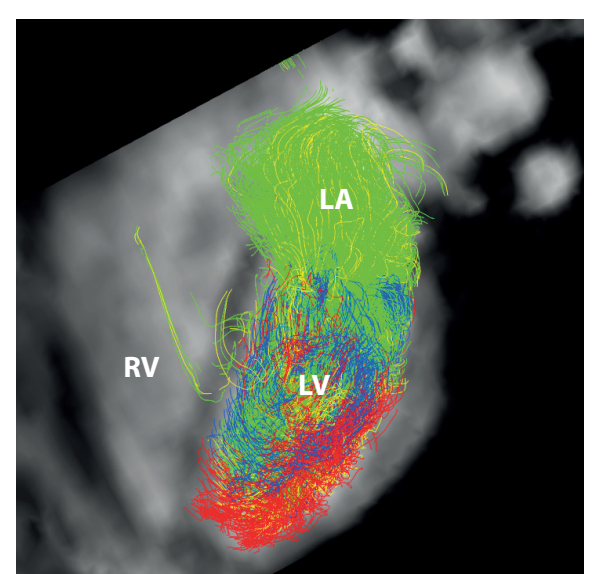

(b) Automatically corrected

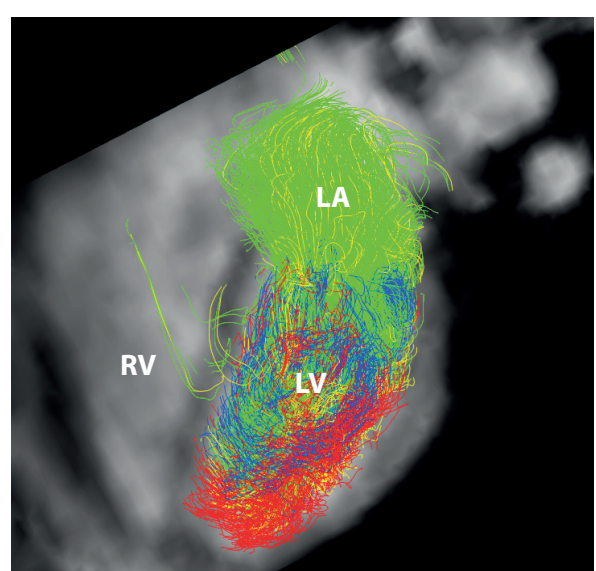

(c) Expert corrected

Figure 5: Outflow (top) and inflow (bottom) pathline analysis performed for the three variants of segmentation: uncorrected, automatically corrected, and expert corrected. The outflow visualization depicts the flow during early systolic ejection from the left ventricle (LV) into the aorta (Ao), while the inflow visualization shows the flow that progresses from the left atrium (LA) into the left ventricle (LV) during ventricular filling. The pathlines' colors correspond to the flow analysis method [23. In the uncorrected segmentations, visible displacements are shown with red arrows. Errors in the flow pathline analysis are shown with white arrows. The flow visualization is overlaid onto a slice of the 4D Flow MRI magnitude image located in a four-chamber view.

more degrees of freedom.

The major advantage of the proposed method is its preclusion of any manual preprocessing or user interaction. Although the application of registration entails setting multiple parameters, the current approach obviates such requirement. This was facilitated by exhaustive a priori leave-one-out experiments, resulting in a selection of parameters specifically suited for the current application. The total automation makes the current technique particularly suitable for clinical use. Other major obstacles the current method overcomes are the intra-observer bias and processing time. Since no parameter needs to be set manually, variation in output is non-existent when applied on the same dataset multiple times. The method requires only 15-20 seconds per slice, and is easily parallelizable.

\section{Limitations}

Poor visibility of the left-ventricular anatomy in the apical region is still challenging, not only for the proposed framework, but also for an experienced clinician. To this end, we used a robust image similarity measure i.e., NMI, which is often used to handle large contrast variations even between multi-modal images. However, as shown in our results, misalignments are still relatively higher around the apex when compared to the other regions of the LV. Our future work aims to address this limitation by further improving the contrast in the apex using the phase contrast variation in $4 \mathrm{D}$ Flow MRI.

\section{CONCLUSION}

Assessment of pulsatile left ventricular blood flow using 4D Flow MRI relies on accurate segmentations of the underlying anatomy. We propose an unsupervised and robust image registration based approach for improving the LV segmentations in 4D Flow MR data using the high contrast in routinely acquired morphological cine-bSSFP images. Evaluation of the proposed method resulted in high 
geometric accuracy when compared with expert corrected segmentations and improvement in LV blood flow analysis compared to using uncorrected segmentations.

\section{ACKNOWLEDGMENTS}

This work was funded by ERC (Heart4flow, Grant: 310612), Swedish Research Council (621-2014-6191), Swedish Heart and Lung Foundation (20140398), and Knut and Alice Wallenberg foundation.

\section{REFERENCES}

1 Dyverfeldt P, Bissell M, Barker AJ, Bolger AF, Carlhäll CJ, Ebbers T, Francios CJ, Frydrychowicz A, Geiger J, Giese D, Hope MD, Kilner PJ, Kozerke S, Myerson S, Neubauer S, Wieben O, Markl M. 4D flow cardiovascular magnetic resonance consensus statement. J Cardiovasc Magn Reson 2015;17(1):72.

2 Bieri O, Scheffler K. Fundamentals of balanced steady state free precession MRI, 2013.

3 McLeish K, Hill DLG, Atkinson D, Blackall JM, Razavi R. A study of the motion and deformation of the heart due to respiration. IEEE Trans Med Imaging 2002;21(9):1142-50.

4 Scott AD, Keegan J, Firmin DN. Motion in cardiovascular MR imaging. Radiology 2009;250(2):331-351.

5 Holland AE, Goldfarb JW, Edelman RR. Diaphragmatic and cardiac motion during suspended breathing: preliminary experience and implications for breath-hold mr imaging. Radiology 1998;209(2):483-489.

6 Svalbring E, Fredriksson A, Eriksson J, Dyverfeldt P, Ebbers T, Bolger AF, Engvall J, Carlh??ll CJ. Altered diastolic flow patterns and kinetic energy in subtle left ventricular remodeling and dysfunction detected by 4D flow MRI. PLoS One 2016;11(8).

7 Fredriksson AG, Svalbring E, Eriksson J, Dyverfeldt P, Alehagen U, Engvall J, Ebbers T, Carlhäll CJ. 4D flow MRI can detect subtle right ventricular dysfunction in primary left ventricular disease. J Magn Reson Imaging 2016;43(3):558-565.

8 Maintz JA, Viergever MA. A survey of medical image registration. Medical Image Analysis 1998;2(1):1-36.

9 Slomka PJ, Fieno D, Ramesh A, Goyal V, Nishina H, Thompson LEJ, Saouaf R, Berman DS, Germano G. Patient motion correction for multiplanar, multi-breath-hold cardiac cine MR imaging. Journal of Magnetic Resonance Imaging 2007;25(5):965973.

10 Elen A, Hermans J, Ganame J, Loeckx D, Bogaert J, Maes F, Suetens P. Automatic 3-D breath-hold related motion correction of dynamic multislice MRI. IEEE Transactions on Medical Imaging 2010;29(3):868-878.

11 Carminati MC, Maffessanti F, Caiani EG. Nearly automated motion artifacts correction between multi breath-hold short-axis and long-axis cine CMR images. Computers in Biology and Medicine 2014;46(1):42-50.

12 Petitjean C, Dacher JN. A review of segmentation methods in short axis cardiac MR images. Med Image Anal 2011;15(2):169184.

13 Xiang QS. Temporal phase unwrapping for CINE velocity imaging. Journal of magnetic resonance imaging : JMRI 1995;5(5):529-34.

14 Ebbers T, Haraldsson H, Dyverfeldt P, Sigfridsson A, Warntjes MJB, Wigström L. Higher order weighted least-squares phase offset correction for improved accuracy in phase-contrast mri. In ISMRM. 2008; .

15 Otsu N. A threshold selection method from gray-level histograms. Automatica 1975;11(285-296):23-27.

16 Klein S, Staring M, Murphy K, Viergever MA, Pluim JP. Elastix: a toolbox for intensity-based medical image registration. IEEE transactions on medical imaging 2010;29(1):196-205.
17 Gupta V, Kirisli HA, Hendriks EA, van der Geest RJ, van de Giessen M, Niessen W, Reiber JH, Lelieveldt BP. Cardiac $\{M R\}$ perfusion image processing techniques: A survey. Medical Image Analysis 2012;16(4):767 - 785 .

18 Thevenaz P, Unser M. Optimization of mutual information for multiresolution image registration. IEEE Trans Image Process 2000;9(12):2083-2099.

19 Qiao Y, Lew B, Lelieveldt B, Staring M. Fast Automatic Step Size Estimation for Gradient Descent Optimization of Image Registration. IEEE Trans Med Imaging 2015;35(2):391-403.

20 Rohlfing T. Image similarity and tissue overlaps as surrogates for image registration accuracy: Widely used but unreliable. IEEE Transactions on Medical Imaging 2012;31(2):153-163.

21 Dice LR. Measures of the Amount of Ecologic Association Between Species. Ecology 1945;26(3):297-302.

22 Hausdorff F. Set Theory. American Mathematical Soc., 1957.

23 Eriksson J, Carlhäll CJ, Dyverfeldt P, Engvall J, Bolger AF, Ebbers T. Semi-automatic quantification of $4 \mathrm{~d}$ left ventricular blood flow. Journal of Cardiovascular Magnetic Resonance 2010; 12(1):1-10.

24 Chandler AG, Pinder RJ, Netsch T, Schnabel JA, Hawkes DJ, Hill DLG, Razavi R. Correction of misaligned slices in multislice cardiovascular magnetic resonance using slice-to-volume registration. J Cardiovasc Magn Reson 2008;10:13.

\section{Supplementary Material}

Supporting Table S1: Dice similarity coefficient (DSC) and Hausdorff distance (HD) measured in all regions of the left ventricle (LV) for the entire cohort. These measures show the accuracy of uncorrected $\left(S_{\text {uncorr }}\right)$ and automatically corrected segmentations, $S_{\text {auto }}$ with respect to the expert corrected segmentations $\left(S_{\text {expert }}\right)$. $S_{\text {auto }}(N M I)$ and $S_{\text {auto }}(N C C)$ were obtained using NCC and NMI as the cost functions, respectively. The value of DSC varies between 0 and 1 , where 1 refers to a complete overlap. The interquartile range (IQR) reports the measures for $25 \%-75 \%$ of the cohort population.

\begin{tabular}{|c|ccc|c|ccc|}
\hline Compared & \multicolumn{3}{|c|}{ Dice similarity coefficient } & & \multicolumn{3}{c|}{ Hausdorff distance (mm) } \\
\cline { 2 - 8 } Segmentations & Mean \pm SD & Median & IQR & & Mean \pm SD & Median & IQR \\
\hline$S_{\text {uncorr vs } S_{\text {expert }}}$ & $0.89 \pm 0.07$ & 0.91 & $0.85-0.93$ & & $3.67 \pm 2.13$ & 3.31 & $2.22-4.44$ \\
$S_{\text {auto }}$ (NMI) vs $S_{\text {expert }}$ & $0.94 \pm 0.02$ & 0.94 & $0.93-0.95$ & & $2.64 \pm 1.08$ & 2.72 & $1.69-2.97$ \\
$S_{\text {auto }}$ (NCC) vs $S_{\text {expert }}$ & $0.87 \pm 0.06$ & 0.88 & $0.84-0.91$ & & $4.27 \pm 2.55$ & 3.54 & $2.70-5.03$ \\
\hline
\end{tabular}

